\title{
Effects of Food Intake on the Mucoadhesive and Gastroretentive Properties of Submicron-Sized Chitosan-Coated Liposomes
}

\author{
Hikaru Sugihara, ${ }^{a}$ Hiromitsu Yamamoto, ${ }^{b}$ Yoshiaki Kawashima, ${ }^{b}$ and Hirofumi Takeuchi*,a \\ ${ }^{a}$ Department of Drug Delivery Technology and Sciences, Laboratory of Pharmaceutical Engineering, Gifu \\ Pharmaceutical University; 1-25-4 Daigaku-Nishi, Gifu 501-1196, Japan: and ${ }^{b}$ Laboratory of Pharmaceutical \\ Engineering, Aichi Gakuin University; 1-100 Kusumoto-cho, Chikusa-ku, Nagoya 464-8650, Japan. \\ Received May 22, 2012; accepted July 23, 2012
}

The gastrointestinal transition of mucoadhesive drug carriers may be affected by food intake, since food changes the physiological conditions of the gastrointestinal tract, and the food content itself is a physical obstruction for the drug carriers. Here we investigated the effects of food intake on the gastrointestinal transition and mucoadhesive function of submicron-sized chitosan-coated liposomes (ssCS-Lip). The stomach and small intestine were removed after oral administration of ssCS-Lip and non-coated liposomes (ssLip) containing fluorescent dye to fasted or fed rats, and retentive properties were quantitatively confirmed by measuring the amount of dye in each part of the gastrointestinal tract. Both types of liposome were retained in the stomach at approx. $40 \%$ in the fed rats at $1 \mathrm{~h}$ after oral administration, whereas transitions in the intestine were reduced compared to the fasted rats. However, the transition of ssCS-Lip in intestine was prolonged compared to ssLip even, in the fed state. The mucoadhesive behavior of ssCS-Lip was evaluated by confocal laser scanning microscopy. The ssCS-Lip tended to penetrate into the mucosal part of the intestine, and in addition, ssCS-Lip was detected in the basolateral side in both conditions, and therefore the mucopenetrative function was confirmed in the fed condition. Based on these results, we confirmed that ssCS-Lip shows a predominant gastrointestinal transition and mucopenetration, even after food intake.

Key words liposome; chitosan; mucoadhesion; food intake; oral

A drug delivered in a mucoadhesive dosage form adheres to mucosa in the gastrointestinal tract. The mucoadhesive dosage form improves the absorption of poorly absorbed compounds based on its extended retention time at absorption sites.

Liposomes are phospholipid vesicles, comprising a phospholipid bilayer surrounding an aqueous compartment. Due to their biphasic characteristic and diversity in design, liposomes offer an adaptable function for improving drug absorption. We developed mucoadhesive liposomes by coating the anionic liposomal surface with a cationic mucoadhesive polymer, chitosan. The effectiveness of the chitosan-coated liposomes (CS-Lip) was confirmed in previous studies that showed enhanced and prolonged pharmacological effects of insulin, which was orally administered to rats in the polymercoated liposomal form. ${ }^{1,2)}$ The effectiveness of mucoadhesive liposomes in drug absorption has also been demonstrated by using calcitonin as a model peptide drug. Carbopol-coated liposomes, with a mucoadhesive property similar to that of CS-Lip, were as effective as CS-Lip. ${ }^{3)}$ In addition, the effects of particle size on the mucoadhesive properties of chitosan-coated liposomes and on the absorption of entrapped calcitonin were evaluated. Submicron-sized chitosan-coated liposomes (ssCS-Lip) showed excellent penetration into the intestinal mucosa, and the pharmacological effect of calcitonin was prolonged up to $120 \mathrm{~h}$ after oral administration to rats. ${ }^{4}$

The influence of food on gastrointestinal conditions should be considered for oral dosage forms. The presence of food can affect the absorption of drugs and may enhance, delay, or reduce absorption. ${ }^{5)}$ Variations in drug absorption may be due to several different effects. Primarily, the effect of food on gastric emptying is considerable, and variations in the rate at which food is presented to the small intestine will change a

The authors declare no conflict of interest. drug's pharmacokinetics. Secondly, a drug can interact with the food in the intestinal lumen, adhere to the food or be or absorbed by it. The presence of viscous chime can act as a physical barrier, reducing drug access to the absorbing surface. ${ }^{6}$

As noted above, drug carriers can aid the absorption of poorly absorbed drugs. However, drug carriers are also affected by food intake, $\mathrm{pH}$, motility and gastric contents. For example, food consumption remarkably reduced the extent of absorption of indomethacin after the oral administration of bioadhesive-containing indomethacin tablets to dogs. ${ }^{7}$ In contrast, when adhesive microspheres containing riboflavin were administered to fed human subjects, drug absorption was improved and the mean residence time was more prolonged compared to when the microspheres were administered to fasted subjects. ${ }^{8)}$ Although these studies' findings clarified that drug absorption is affected by food intake, they did not evaluate the behavior of the carrier itself. Changes in the mucoadhesive effects of drug carriers on food intake have not been sufficiently established.

In the present study, the effect of feeding on the gastrointestinal transition and mucoadhesive behavior of ssCS-Lip was evaluated quantitatively and visually by confocal laser scanning microscopy (CLSM) in order to precisely clarify the carrier function in food intake.

\section{Experimental}

L- $\alpha$-Distearoylphosphatidylcholine (DSPC; Nippon Oil and Fats Co., Tokyo), dicetylphosphate (DCP; Sigma-Aldrich, St. Louis, MO, U.S.A.), cholesterol (Chol; Sigma-Aldrich) and 1,1'-dioctadecyl-3,3,3',3'-tetramethylindo carbocyanine perchlorate (DiI; Lambda, Rainbach, Austria) were used as received. Chitosan (CS), which is a deacetylated chitin (poly- $N$-deacetylglucosamine) industrially prepared by hydrolyzing the aminoacetyl groups of chitin in aqueous alkaline 
solution, was a gift from Katakura-Chikkarin Co. (Tokyo). The molecular weight and the deacetylation percentage of the CS used were approx. 150000 and 85\%, respectively. All other reagents were of analytical grade.

Preparation of ssCS-Lip The preparation of the submicron-sized chitosan-coated liposomes was described in detail previously. ${ }^{2)}$ Anionic multilamellar liposomes (MLV) consisting of DSPC, DCP and Chol in a molar ratio of $8: 2: 1$ and containing DiI were prepared using the thin-film hydration method. In a typical procedure, $161 \mathrm{mg}(204 \mu \mathrm{mol})$ of DSPC, $27.9 \mathrm{mg}(51 \mu \mathrm{mol})$ of DCP, $9.7 \mathrm{mg}(25 \mu \mathrm{mol})$ of Chol, and $1.68 \mathrm{mg}$ of DiI were dissolved in a small amount of chloroform, and the solution was rotary-evaporated at $40^{\circ} \mathrm{C}$ to obtain a thin lipid film. The thin lipid film was dried in a vacuum oven overnight to ensure complete removal of the solvent, and hydration was carried out with $5 \mathrm{~mL}$ of acetate buffer solution ( $\mathrm{pH} 4.4,100 \mathrm{~mm}$ ) by vortexing, followed by incubation at $10^{\circ} \mathrm{C}$ for $30 \mathrm{~min}$. The submicron-sized liposomes (ssLip) were prepared by sonication (Sonifier 250, Branson/Emerson, St. Louis, MO, U.S.A.) the MLV. The particle size of the ssLip was controlled by adjusting the sonication time. For example, ssLip of approx. $200 \mathrm{~nm}$ were prepared by sonicating three times for $3 \mathrm{~min}$ each time. The particle size of the resultant liposomes was confirmed by dynamic light scattering analysis with an LPA-300 photon correlation spectroscopy system (Otsuka Electronics, Osaka, Japan). For the measurement of the liposomal diameters, an aliquot of each liposomal suspension was diluted with a large amount of purified water.

For the preparation of CS-Lip, an aliquot of the liposome suspension was mixed with the same volume of acetate buffer solution $(\mathrm{pH} 4.4)$ of $\mathrm{CS}(0.6 \%)$, followed by incubation at $10^{\circ} \mathrm{C}$ for $1 \mathrm{~h}$. Thus, the final concentration of the lipids and polymers were half of those of the original solutions. The noncoated liposomes were mixed with the corresponding buffer solutions to adjust the liposomal concentrations to those of the coated liposomes. ssCS-Lip were prepared in the same manner. The average particle size of the resultant ssCS-Lip was in the range of $247-320 \mathrm{~nm}$; that of the original noncoated liposomes was $180-200 \mathrm{~nm}$.

Retentive Profile and Observation of the Mucoadhesive Behavior of Liposomal Particles in the Intestine The liposomes containing DiI were administered intragastrically to 13 -week-old rats that had been fasted for $48 \mathrm{~h}$ (defined as "fasted") or fasted for $6 \mathrm{~h}$ (defined as "fed") prior to administration. In the fed rats, feed was given simultaneously with the administration of liposomes. Charles River Formula (CRF)-1 (Oriental Yeast Co., Ltd., Tokyo, Japan) was used as a basal diet throughout the study. The dose was determined based on the amount of DiI formulated to the liposomes; i.e., $0.168 \mathrm{mg}$ $\mathrm{DiI} / \mathrm{rat}$. The stomach and small intestine were excised from rats sacrificed 1,2 , or $4 \mathrm{~h}$ after liposome administration, and the small intestine was divided into five $15-\mathrm{cm}$ segments referred to as "duodenum," "upper or lower jejunum," and "upper/lower ileum." Each part of the gastrointestinal tract was homogenized with $3 \mathrm{~mL}$ of saline solution without washing. DiI in the homogenate $(0.3 \mathrm{~mL})$ was extracted with $6 \mathrm{~mL}$ of a mixed organic solvent of chloroform and methanol $(1: 1)$ by shaking for $40 \mathrm{~min}$. The DiI concentration in the organic solvent separated from the mixture by centrifugation (3500 rpm, 10 min) was measured with fluorometry (F3010, Hitachi, Tokyo) at an excitation wavelength of $550 \mathrm{~nm}$ and an emission wavelength of $570 \mathrm{~nm}$.

For the observation of the mucoadhesive behavior of the liposomes, the small intestine was excised from rats sacrificed $1 \mathrm{~h}$ after liposome administration and then washed with a large amount of saline solution (approx. $20 \mathrm{~mL}$ ). Each part of the rat intestine was sliced with a cryostat (Leica, Wetzlar, Germany) to generate sections for confocal laser scanning microscopy (LSM510, Carl Zeiss Microscopy Co., Tokyo) and observed at an excitation wavelength of $550 \mathrm{~nm}$ and emission wavelength of $570 \mathrm{~nm}$.

\section{Results and Discussion}

Retentive Profile of ssCS-Lip The retentive profiles of ssCS-Lip and ssLip in the rat gastrointestinal tract were evaluated by measuring the amount of particles after oral administration. ssCS-Lip and ssLip containing fluorescent dye were administered to fasting or fed rats, and then the gastrointestinal tracts were removed $1 \mathrm{~h}$ after administration. As shown in Fig. 1, the transitions of ssLip at the lower jejunum and the ileum were predominant in the fasted rats, whereas retention in the intestinal tract in the fed rats was reduced. In contrast, $44.7 \%$ of ssLip remained in the stomach after administration of ssLip to the fed group. The ssCS-Lip group's transition showed a similar tendency. The ssCS-Lip was mainly distributed at the jejunum and the upper ileum in the fasted group; however, ssCS-Lip was distributed at the lower jejunum and ileum in the fed rats and was retained at $40.9 \%$ in the stomach. Although the intestinal residence of both types of liposomes in the fed rats was decreased, ssCS-Lip was significantly retained in the upper and lower ileum compared to ssLip. The recovery of ssLip - as a sum of the stomach and each part of the intestinal tract-was $59.5 \%$, indicating that approx. $40 \%$ moved to the large intestine. The recovery of ssCS-Lip was $90.2 \%$, significantly higher than that of ssLip, revealing that the gastrointestinal transition of ssCS-Lip was prolonged by the contribution of the mucoadhesive function, even in the fed state.

In the fed rats, both types of liposome were markedly retained in the stomach. Although the stomach functions to digest food, gastric emptying was prevented by the food intake in the fed rats, and thus we observed significant amounts of both types of liposome in the stomach.

In a study with dogs, food consumption markedly reduced the extent of absorption of indomethacin after the oral administration of bioadhesive-containing indomethacin tablets. ${ }^{7)}$ The most plausible explanation for this decrease in bioavailability is the complexation of the bioadhesive material with a dietary component. In the present study's comparison of ssCS-Lip and ssLip, the retentive profiles were reduced in the fed state; however, ssCS-Lip was more retentive than ssLip. Therefore, the changes in the gastrointestinal distributions in the fed group seemed to be caused by the promotion of gastrointestinal motility, not by adhesion to the food component or the lack of mucoadhesion.

Time Dependency of Liposomal Distribution in the Gastrointestinal Tract In our laboratory we have quantitatively evaluated the time dependency of the gastrointestinal transition of ssCS-Lip and ssLip in fasted rats, and we observed that ssCS-Lip showed extended transition in the gastrointestinal tract due to its mucoadhesive function. ${ }^{9)}$

In the present study, therefore, we also investigated the 


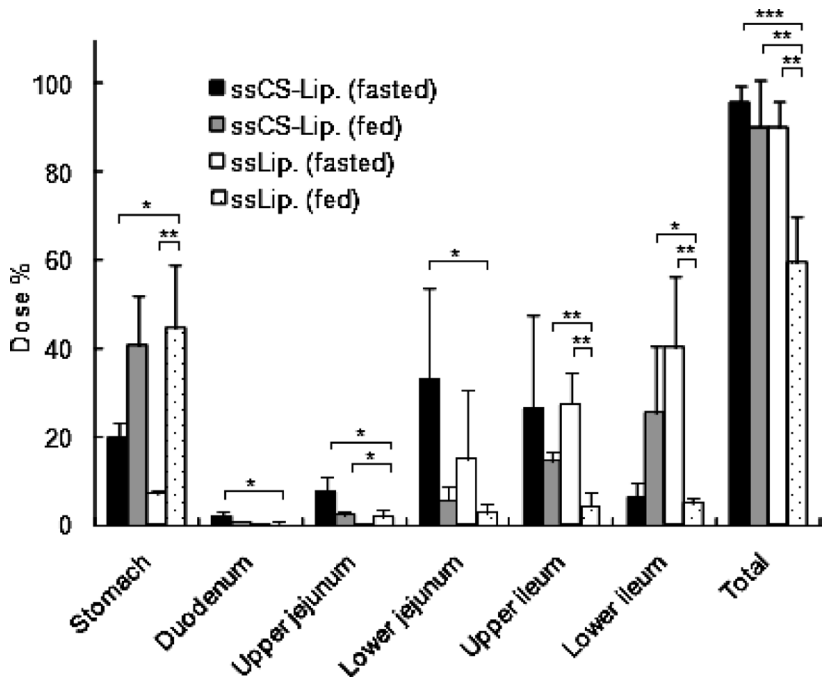

Fig. 1. Effect of Feeding on the Gastrointestinal Transition of ssCS-Lip and ssLip in Various Parts of the Gastrointestinal Tract at $1 \mathrm{~h}$ after Intragastric Administration

The measured mean particle sizes of ssCS-Lip (fasted), ssCS-Lip (fed), ssLip (fasted) and Lip (fed) were 304.7, 301.3, 182.9, and $198.4 \mathrm{~nm}$, respectively. The formulation of the liposomes was DSPC: DCP:Chol $=8: 2: 1$. The concentration for coating was $0.3 \%$. $* p<0.05, * * p<0.01, * * * p<0.001$ : significantly different from ssLip (fed). ( $n=4$ for each group.)

effect of feeding on the time dependency of the gastrointestinal transition of ssCS-Lip. The liposomal distributions following the oral administration of ssCS-Lip in the fasting and fed conditions are shown in Fig. 2. The transition at the lower jejunum and upper ileum was predominant at $1 \mathrm{~h}$ in the fasted group, whereas the liposomes migrated to the lower ileum in the fed group. The intestinal transition of liposomes showed a similar tendency at 2 and $4 \mathrm{~h}$ after administration. The intestinal transition of ssCS-Lip was reduced in the fed group.

Although the retentive percentages of ssCS-Lip in the stomach in the fasting and fed groups were $19.9 \%$ and $40.9 \%$, respectively, the retentive amount was significantly increased in the fed group $1 \mathrm{~h}$ after liposome administration. Similar results were observed at 2 and $4 \mathrm{~h}$ after liposome administration. The liposomal transitions in the stomach at $2 \mathrm{~h}$ in the fasted and fed groups were $7.3 \%$ and $28.3 \%$, and were $3.1 \%$ and $11.5 \%$ at $4 \mathrm{~h}$, respectively. The total percentages of liposomes retained in the gastrointestinal tract at $2 \mathrm{~h}$ in the fasting and fed groups were $96.0 \%$ and $60.4 \%$, and were $38.1 \%$ and $22.5 \%$ at $4 \mathrm{~h}$, respectively. In light of these results, we see that although the liposomes moved rapidly through the intestinal tract, a marked level of liposomes remained in the stomach in the fed rats.

Observation of Mucoadhesive Properties One of the functions of mucosa is to restrict the penetration of large molecules, including particles. It was thought that particles rarely pass through the absorptive enterocytes of mucosa. However, when we we observed the rat intestinal tract with CLSM, ${ }^{4}$ we found that submicron-sized liposomal particles penetrated into the mucosa, while such behavior was not observed for approx. 3- $\mu \mathrm{m}$ multilamellar liposome particles. In the present study, therefore, we evaluated the mucopenetrative functions of ssCS-Lip in non-fasted rats.

The liposomal distributions in the fed vs. fasting states in the intestinal tract were evaluated by quantifying the residual liposomes on the mucosa with CLSM, as shown in Fig. 3.
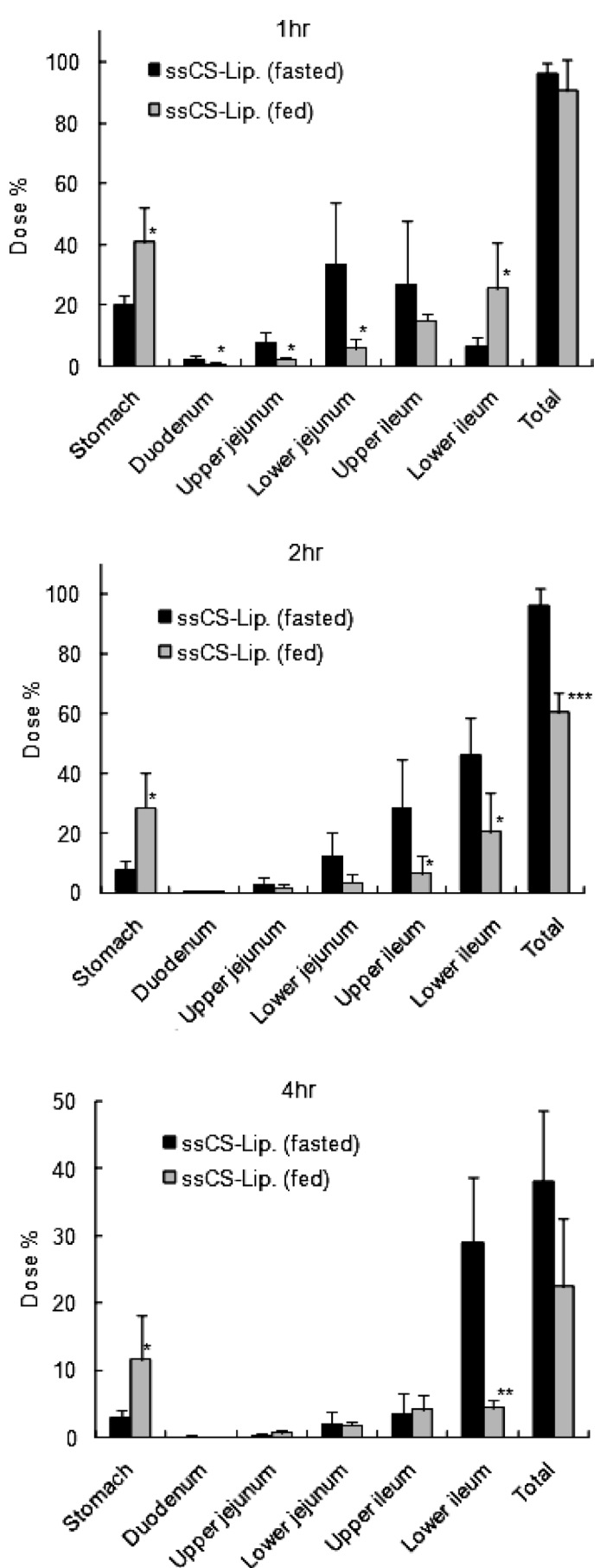

Fig. 2. Effect of Feeding on the Gastrointestinal Transition of ssCS-Lip in Various Parts of the Gastrointestinal Tract at 1, 2, and $4 \mathrm{~h}$ after Intragastric Administration

The measured mean particle sizes of ssCS-Lip (fed) and (fasted) were 301.3 and $304.7 \mathrm{~nm}$ at $1 \mathrm{~h}, 265.7$ and $304.7 \mathrm{~nm}$ at $2 \mathrm{~h}$, and 247.4 and $270.6 \mathrm{~nm}$ at $4 \mathrm{~h}$, respectively. The formulation of the liposomes was DSPC:DCP :Chol=8:2:1. The concentration for coating was $0.3 \%$. $* p<0.05, * * p<0.01$, *** $p<0.001$ : significantly different from ssCS-Lip (fasted). ( $n=4$ for each group.)

The white lines in each photograph show the edges of sliced intestinal membrane, and the red parts suggest the existence of liposomes. Comparing the images from fasted and fed rats, ssCS-Lip was detected at the lower ileum in the fed group. The liposomal distribution in the images and the quantitative evaluation showed a similar pattern.

Our observations of the mucus layer revealed considerable amounts of liposome throughout the intestinal tract, and the 


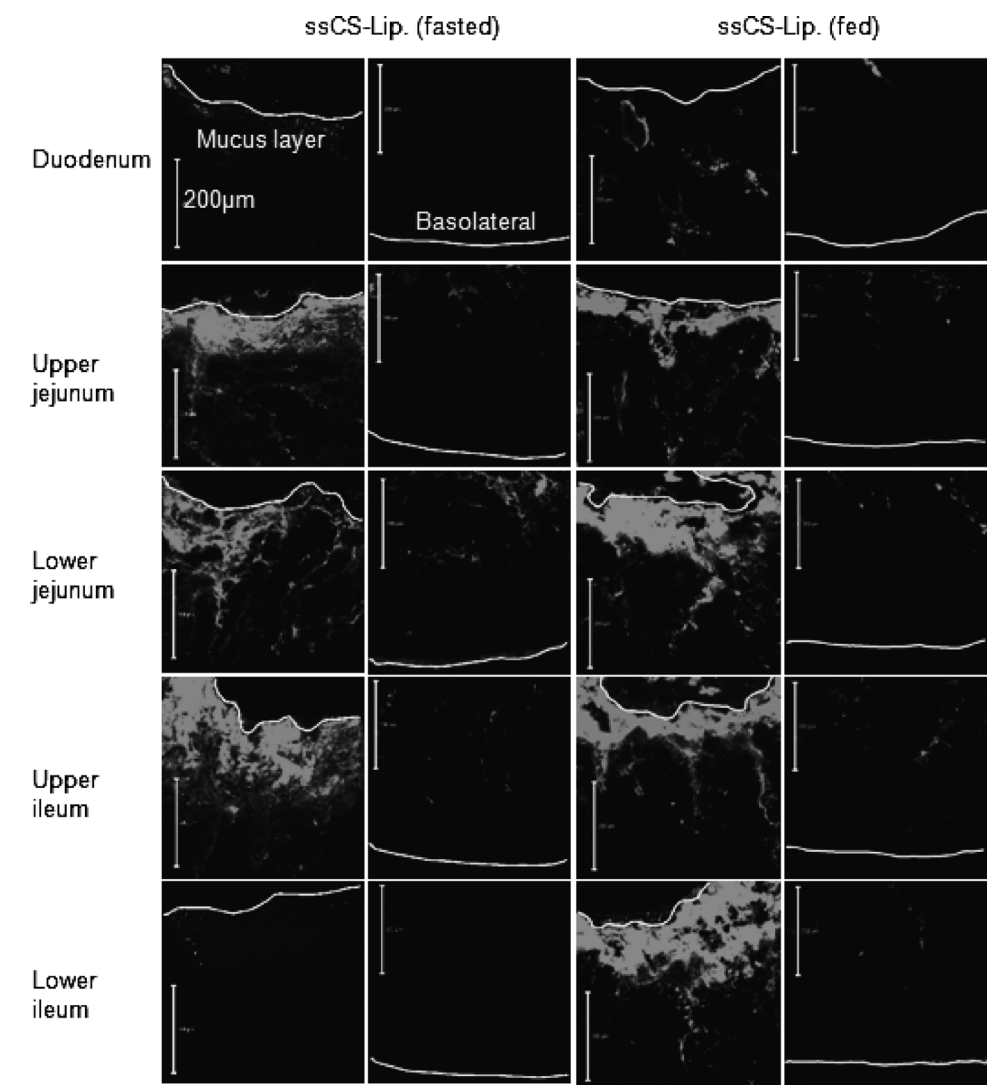

Fig. 3. Confocal Laser Scanning Microscopic Images of ssCS-Lip in Various Parts of the Intestinal Tract at $1 \mathrm{~h}$ after Intragastric Administration under Fasted and Fed Conditions

The measured mean particle sizes of ssCS-Lip (fasted) and (fed) were 320.2 and $290.3 \mathrm{~nm}$, respectively. The formulation of the liposomes was DSPC:DCP:Chol=8:2:1. The concentration for coating was $0.3 \%$.

liposomes tended to penetrate into the mucosal part of the intestine. We also observed liposomes in the basolateral side of the intestinal membrane in the fed group as well as the fasted group. Based on these observations, we note that ssCS-Lip possesses a similar penetrative property in the fed condition, although its retention property was lower than in the fasting condition.

Previous studies demonstrated particle uptake in the gastrointestinal tract or Caco-2 cells. Jani and colleagues showed the absorption of polystyrene nanoparticles and confirmed that their uptake occurred through both gut-associated lymphoid tissue (GALT) and, to a lesser extent, normal intestinal tissue. ${ }^{10-12)}$ One of the possible mechanisms explaining the uptake of ssLip in the present study is adsorptive endocytosis, as the GALT (Peyer's patches) was excluded in the preparation of the samples for CLSM observation. The particles must pass through the mucus layer before reaching the surface of enterocytes. An interaction of chitosan on the surface of ssCS-Lip with mucin may contribute to pass through the mucus layer. Although more experiments are needed to establish the absorption mechanism of liposomal particles, adsorptive endocytosis is the most likely mechanism.

\section{Conclusion}

The effect of food intake on the mucoadhesive properties of ssCS-Lip was evaluated quantitatively and qualitatively. Although the rat intestinal transition of ssCS-Lip was affected in the fed state, gastric transition was markedly prolonged. In addition, ssCS-Lip showed an excellent mucopenetrative property in the fed state as well as the fasting state. Based on these results, we confirmed that ssCS-Lip shows a predominant gastrointestinal transition even after food intake.

Acknowledgement We are grateful to Katakura-Chikkarin Co. for supplying chitosan.

\section{References}

1) Takeuchi H., Yamamoto H., Niwa T., Hino T., Kawashima Y., Chem. Pharm. Bull., 42, 1954-1956 (1994).

2) Takeuchi H., Yamamoto H., Niwa T., Hino T., Kawashima Y., Pharm. Res., 13, 896-901 (1996).

3) Takeuchi H., Matsui Y., Yamamoto H., Kawashima Y., J. Controlled Release, 86, 235-242 (2003).

4) Takeuchi H., Matsui Y., Sugihara H., Yamamoto H., Kawashima Y., Int. J. Pharm., 303, 160-170 (2005).

5) Toothaker R. D., Welling P. G., Annu. Rev. Pharmacol. Toxicol., 20, 173-199 (1980)

6) "Physiological Pharmaceutics: Barriers to Drug Absorption," 2nd ed., ed. by Washington N., Washington C., Wilson C. G., Taylor \& Francis, New York, 2001, p. 133.

7) Hosny E. A., El-Sayed Y. M., Al-Meshal M. A., Al-Angary A. A., Int. J. Pharm., 112, 87-91 (1994).

8) Akiyama Y., Nagahara N., Nara E., Kitano M., Iwasa S., Yamamoto I., Azuma J., Ogawa Y., J. Pharm. Pharmacol., 50, 159-166 (1998).

9) Sugihara H., Yamamoto H., Kawashima Y., Takeuchi H., J. Lipid Res., 22, 72-79 (2012).

10) Jani P. U., Halbert G. W., Langridge J., Florence A. T., J. Pharm. Pharmacol., 42, 821-826 (1990).

11) Hillery A. M., Jani P. U., Florence A. T., J. Drug Target., 2, 151156 (1994).

12) Florence A. T., Pharm. Res., 14, 259-266 (1997). 